\title{
La calidad, valor diferencial de la televisión informativa en el nuevo mercado de la comunicación: el caso de Colombia
}

\author{
Fecha de recepción: 15 de septiembre de 2011 - Aceptación: 10 de octubre de 2011
}

\section{Juan Camilo Hernández-Rodríguez - Juan Carlos Gómez-Giraldo Liliana María Gutiérrez-Coba - Germán Antonio Arango-Forero}

\section{Resumen}

Los telediarios que triunfan, en el voraz mercado de la información, son aquellos que reconocen en la calidad periodística un parámetro diferencial de competitividad y, por tanto, su más importante fuente de rentabilidad. Desafortunadamente en Colombia los noticieros de la televisión abierta no le otorgan importancia a la investigación propia, a la consulta diversificada de fuentes, a la búsqueda de un equilibrio informativo y al uso de elementos de contexto; todos, componentes de calidad; tal como se evidencia en este artículo, que recoge una parte de los hallazgos de una observación sistemática a estos tele informativos que elaboró el Observatorio de Medios de la Facultad de Comunicación de la Universidad de La Sabana y financiada por la Comisión Nacional de Televisión.

\section{Abstract}

The television news bulletins that succeed in the voracious market of information are those that acknowledge a differential parameter of competitiveness within journalistic quality; therefore, it becomes its most important source of profitability. Unfortunately, in Colombia the news that belongs to open television does not give importance to own research, to diversified inquiry of sources, to the search for an informative balance, or to the use of context elements, all of which are quality components. All this is evidenced in this article, which also shows part of the findings of a systematic observation made to this news by the Media Observatory of the Faculty of Communication belonging to the Universidad de La Sabana and financed by the National Commission of Television.

\section{Palabras clave}

Televisión, noticieros, información, calidad, mercado, diferencial de competitividad.

\section{Keywords}

Television, News, Information, Quality, Market, Competitiveness Differential

\section{Introducción}

La información, a pesar de ser algo intangible, es una de las materias primas más valiosas en el mercado global, y centro de disputa entre diversos actores que reconocen en su dominio el "control de la formación y, en definitiva, el control del poder" (Monedero, 2009:34). Es tal su importancia que desde hace varios años se habla de una era e, incluso, de una sociedad de la información (Castells, 2000; Matterlart, 2002). 
El debate sobre lo acertado o inocuo de mercantilizar la información puede tornarse todavía más sensible al centrar la atención en uno de los mayores promotores $y$ exponentes de la sociedad de la información: la televisión, cuyos contenidos no escapan, al igual que no lo hacen los del resto de los medios de comunicación, a las lógicas de la hipermodernidad, donde prima "la cultura del más aprisa y el siempre más: más rentabilidad, más eficacia, más ductilidad, más innovación"
Por desgracia, según sugieren autores como Isidro Catela (2006), el encumbramiento de dicha sociedad ha contagiado a la comunicación de las maneras de la economía, convirtiéndola en objeto de compra y venta; o sea, sometida a unas leyes: las de oferta y demanda. Impregnada, entonces, de las lógicas del mercado, la información se reduce al estado de mercancía.

Lo peligroso de esta concepción es que, al imbuir por ejemplo a las noticias en el frenesí consumista, "los acontecimientos noticiosos

\section{Reseña de autores \\ Juan Camilo Hernández-Rodríguez (Colombia) \\ Universidad de la Sabana \\ juan.hernandez6@unisabana.edu.co \\ Profesor del Área de Fundamentación y Contexto de la Facultad de Comunicación de la Universidad de La Sabana y Coordinador Académico del Observatorio de Medios.}

\section{Juan Carlos Gómez-Giraldo (Colombia) \\ Universidad de la Sabana \\ juan.gomez@unisabana.edu.co \\ Doctorando en Ciencias de la Comunicación de la Universidad Austral de Argentina. Profesor del Área de Fundamentación y Contexto de la Facultad de Comunicación de la Universidad de La Sabana e inves- tigador del Observatorio de Medios.}

\section{Liliana María Gutiérrez-Coba (Colombia) \\ Universidad de la Sabana \\ liliana.gutierrez1@unisabana.edu.co \\ Doctora en Ciencias de la Información por la Universidad del País Vasco (España). Profesora del Área de Periodismo de la Facultad de Comunicación de la Universidad de La Sabana y Directora del Grupo de Investigación en Periodismo (GIP).}

\section{Germán Antonio Arango-Forero (Colombia)}

Universidad de la Sabana

german.arango@unisabana.edu.co

Doctorando en Ciencias de la Comunicación de la Universidad Austral de Argentina. Profesor del Área de Audiovisual de la Facultad de Comunicación de la Universidad de La Sabana e investigador del Observatorio de Medios. que no tienen fácil venta quedan discriminados, y de ello resulta una presentación distorsionada de la actualidad, a costa de hacerla más comercial" (Dader, 2009: 155).

$Y$ es que si se acepta entender la noticia como producto de consumo, tendría que hacerse la salvedad que no se trata de uno cualquiera, porque este es un "producto social" (Gouldner, 1976; Lippmann, 1965) o una reconstrucción de la realidad. De ahí que los informativos posean "una poderosa capacidad de influencia en la opinión pública a través de la imagen que presentan acerca de la realidad política, económica, social, cultural, etc." como afirma José Alberto García (2007).

El debate sobre lo acertado o inocuo de mercantilizar la información puede tornarse todavía más sensible al centrar la atención en uno de los mayores promotores y exponentes de la sociedad de la información: la televisión, cuyos contenidos no escapan, al igual que no lo hacen los del resto de los medios de comunicación, a las lógicas de la hipermodernidad", donde prima "la cultura del más aprisa y el siempre más: más rentabilidad, más eficacia, más ductilidad, más innovación" (Dader, 2009:150).

No obstante, desconocer el grado de permeabilidad que ha tenido el mercado y su mismo raciocinio sobre el mundo de la comunicación es navegar contracorriente. Además, es posible que se esté desechando la oportunidad de encontrar en las dinámica propias del consumo un sinfín de referentes para justificar y exigir la imperiosa presencia de valores antropológicos y morales en la producción de información y, más exactamente, en la manera de hacer

1. El término es de Gilles Lipovetsky y con éste describe un estadio avanzado de la vieja Modernidad. Para Lipovetsky (citado por Dader: 2009: 150), en la Hipermodernidad "proliferan las patologías individuales, el consumo desmedido y los comportamientos anárquicos". 
televisión informativa-centro de interés de este artículo-.

Fíjese que la primera línea de orientación está dada por el siguiente interrogante que se hace cualquier empresa en la actualidad: ¿cómo abrirse paso en el mundo de la oferta y la demanda? Aplicada a nuestro caso: ¿a qué estrategias recurren las empresas informativas para ganarse el favor del ciudadano-espectador, es decir, de su cliente?

Como sucede con cualquier producto, las noticias entregadas por uno y otro oferente televisivo deben poseer unas "ventajas comparativas que le confieran un diferencial de competitividad sobre sus contendientes inmediatos" (cfr. Pérez, 2008: 390). Al respecto, parece existir un acuerdo entre todos los sectores productivos, del que no escapa el mundo del periodismo, sobre un concepto que expresaría la excelencia de un producto sobre otro: la calidad. Un vocablo no menos complejo de abordar, dado su polivalente carácter, es el denominado concepto de calidad, pues se habla de calidad de bienes y servicios, calidad de vida, calidad humana y hasta de una gestión total de la calidad.

Sin embargo, el estudio de la calidad en el campo informativo o periodístico ya ha sido referenciado en buena medida por importantes teóricos, pero sin obtener un consenso sobre su definición o concepción última, aunque han logrado importantes avances a la hora de construir sistemas para la medición de la misma.

Para los fines del presente artículo, que examinará el tratamiento de la información trasmitida por los noticieros colombianos de televisión abierta durante la franja prime time, se entenderá que la calidad en el ámbito periodístico trasciende el plano técnico y exalta sobre todo el rigor y la moral.

Por ello, la definición más pertinente viene de Ángel Benito (2001:27) al explicar que "existe una calidad formal -la imagen, el plano, el sonido, etc.- que hoy la tecnología hace posible casi hasta la perfección máxima, y una calidad de tipo moral, en razón del respeto a la verdad y objetividad posible, selección de contenidos y recto comportamiento profesional".

Una noticia de calidad dependerá, entonces, de que el periodista sea cuidadoso, veraz, equitativo, destaque lo importante y se ponga en el lugar del televidente (Tuggle, 2001). "Pero al mismo tiempo se espera que los buenos productos informativos, entre otras cosas, reflejen la comunidad a la que pertenecen y establezcan con ella un alto grado de conexión" (De la Torre y Téramo, 2007).

En la línea de Téramo y De la Torre, la calidad periodística se traduce en la búsqueda incesante del periodista por optimizar sus procesos de selección y creación de la noticia, etapas que deben superar la simple mejora técnica y adoptar mayor apego a la verdad. De esta manera, en el transcurso del artículo se analizarán sólo algunos indicadores que colaborarían en esta tarea: origen de las noticias, la consulta de fuentes, el equilibrio informativo y el uso de elementos de contexto.

Se reconoce en los anteriores aspectos, por tanto, unos componentes básicos para la consecución de lo que llama Rafael A. Pérez, un diferencial de competitividad o, sin duda, la anhelada calidad que, cabe advertir, se ha convertido en un "caballito de batalla", pues en su descuidado empleo ya no se "reconoce ninguna verdad, pues hay que fingir el acuerdo” (Catela, 2005).

\section{Método}

Bajo el patrocinio de la Comisión Nacional de Televisión (CNTV) y la coordinación de la Asociación Colombiana de Universidades (Ascun), el Observatorio de Medios (OM) de la Facultad de Comunicación de la 
Universidad de La Sabana, en cooperación con el Centro Multimedial (CM) de la Universidad EAFIT de Medellín, desarrollaron en 2010 la investigación Análisis de la estructura, cobertura y contenidos de los noticieros de la televisión colombiana. Para la realización del estudio, se tomó una muestra de 15 noticieros, distribuida de la siguiente manera: 1 noticiero local (el único de televisión abierta existente en el país), 9 regionales y 5 nacionales. En total fueron analizadas 3857 notas periodísticas, contenidas en 154 emisiones de la franja prime time (7:00 p.m. a 11:00 p.m.) del 16 al 30 de octubre de 2009 (cuadro 1).

Tabla 1. Distribución de la muestra de estudio

\begin{tabular}{|c|c|c|c|}
\hline \multicolumn{4}{|c|}{ Universo de noticieros analizados } \\
\hline Canales & Noticieros & No. Emisiones & No. Notas \\
\hline \multicolumn{4}{|c|}{ Noticiero local (Bogotá) } \\
\hline City TV & City Noticias & 15 & 384 \\
\hline \multicolumn{4}{|l|}{ Noticieros regionales } \\
\hline Canal Capital & Capital Noticias & 15 & 257 \\
\hline Teleantioquia & $\begin{array}{l}\text { Teleantioquia } \\
\text { Noticias }\end{array}$ & 12 & 387 \\
\hline \multirow[t]{2}{*}{ Telecafé } & Telecafé Noticias & & \\
\hline & 1 ANoticias & 10 & 22 \\
\hline $\begin{array}{l}\text { Televisión Regional } \\
\text { de Oriente }\end{array}$ & TRO Noticias & 12 & 188 \\
\hline Telecaribe & CV Noticias & 6 & 180 \\
\hline Canal 13 & Canal 13 Noticias & 11 & 186 \\
\hline \multicolumn{4}{|l|}{ Noticieros nacionales } \\
\hline Canal Caracol & Caracol Noticias & 15 & 404 \\
\hline Canal RCN & Noticias RCN & 15 & 426 \\
\hline \multirow[t]{3}{*}{ Canal Uno } & Tele País & 11 & 415 \\
\hline & CM\& & 11 & 423 \\
\hline & Noticias Uno & 4 & 112 \\
\hline Totales & 15 & 154 & 3857 \\
\hline
\end{tabular}

La investigación tiene tres vertientes intelectuales que sustentan las variables y categorías de análisis, según algunas de las más recientes tesis académicas y la experiencia de organismos e instituciones interesados en cuantificar la calidad de la televisión regional, local y nacional.

La primera es la fórmula de Valor Agregado Periodístico (VAP), sistema métrico de calidad informativa creado hace dos décadas por un equipo de investigadores de la Facultad de Comunicaciones de la Universidad Católica de Chile (Alessandri et ál., 2001), validado en diferentes países de América Latina. A través de este modelo se han determinado criterios medibles de calidad periodística que permiten establecer las características de la información publicada por un medio de comunicación y valorarlas.

Un segundo referente para el OM y el CM es la invaluable experiencia del Observatorio de la Televisión de la Universidad Austral en Argentina (Delbosco et ál., 2006), que se ha preocupado por identificar específicamente la calidad de la información televisiva. Desde esta perspectiva, la investigación empleó parte de sus lineamientos en materia de caracterización de los actores del proceso informativo y del lenguaje usado por los mediadores.

Una tercera postura teórica es la teoría de la Agenda-setting (McCombs y Shaw, 1972), modelo científico ampliamente difundido en el mundo y que estudia cómo los medios ejercen influencia en las audiencias mediante los temas considerados de mayor relevancia. Gracias a los conceptos de esta teoría se logró sistematizar el impacto de los noticieros colom- 
bianos en la agenda pública, se pudo medir el enfoque de la noticia (framing), y la forma en la que se presenta el primer encuentro de los televidentes con un tema determinado (priming).

\section{Resultados}

Hechizados por la fuente: sobre el origen de la información

Las noticias no llegan por sí solas a los escritorios de los periodistas; éstas básicamente son fruto bien del ejercicio diario del comunicador al salir a la calle en búsqueda de las historias o del suministro de sucesos por parte de las fuentes interesadas.

En esta dirección, la investigación del OM y el CM fue reveladora: el 34\% de las notas publicadas por los noticieros de televisión parte de la iniciativa de la fuente. Es decir, para dar la información, el periodista ha sido convocado a una rueda de prensa o ha recibido un comunicado en el que la fuente interesada anuncia o da a conocer algo que desea divulgar. El origen de otro $24 \%$ corresponde a registros informativos o al cubrimiento de hechos que ocurren súbitamente y que por su por su carácter noticioso no pueden ser ignorados por un medio de comunicación, tal como sucede con los accidentes de tránsito o los desastres naturales.

Lo anterior da como resultado que un $58 \%$ de los temas que se publican en los telediarios colombianos no son de iniciativa del medio, y, por tanto, son registrados casi en igualdad de condiciones por todos los medios de comunicación. Los temas propuestos por iniciativa de los telediarios son apenas del 17\% (ver gráfico 1).

\section{Gráfico 1. Origen de la información}

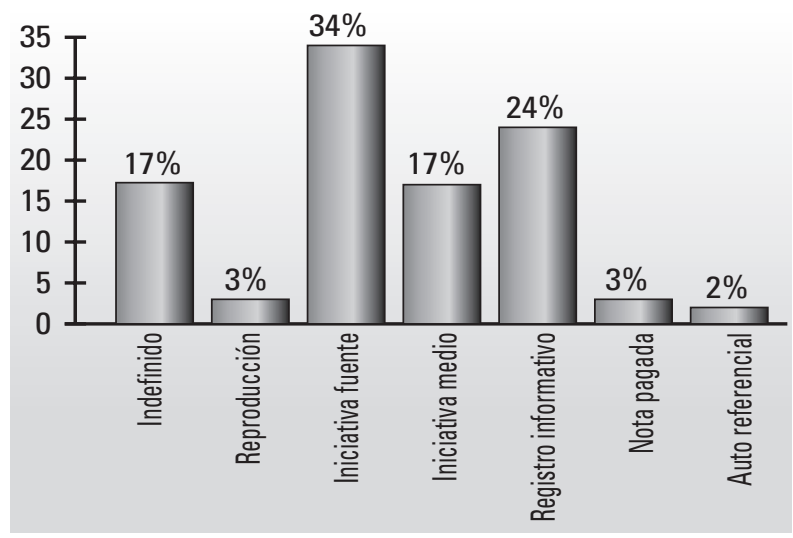

Vale la pena destacar que si bien no es numéricamente representativo el fenómeno, se evidencia el uso de notas auto referenciales (2\%) -o que hacen alusión a productos del mismo medio-y notas pagadas (3\%) dentro de los diversos noticieros.

Si se quisiera hacer una mirada más en detalle de los fenómenos aquí mencionados, se concluiría que en los informativos de carácter nacional, la originalidad reclamada por la teoría del “deber ser” es aún menor. Las notas por iniciativa del medio apenas alcanzan el 11\%, mientras que los contenidos por iniciativa de la fuente o por registro informativo llegan juntas al 50\% (ver tabla 2).

En el ámbito regional, la cantidad de la información obtenida por los reporteros en ruedas de prensa y a través de comunicados suministrados por las fuentes es del 44\%; es decir, un porcentaje muy superior al promedio general (34\%).

Por su parte, el canal local de Bogotá (City TV) mantiene la prelación a la información, cuya iniciativa es de la fuente (38\%) y en segundo grado de importancia, aparecen los temas de iniciativa propia con el $27 \%$. 
Tabla 2. Origen de la información

\begin{tabular}{|l|c|c|c|}
\hline Origen & $\begin{array}{c}\text { Noticieros } \\
\text { nacionales }\end{array}$ & $\begin{array}{c}\text { Noticieros } \\
\text { regionales }\end{array}$ & $\begin{array}{c}\text { Noticiero } \\
\text { Local }\end{array}$ \\
\hline Indefinido & $28 \%$ & $8 \%$ & $3 \%$ \\
\hline Reproducción & $4 \%$ & $3 \%$ & $4 \%$ \\
\hline Iniciativa de la fuente & $24 \%$ & $44 \%$ & $38 \%$ \\
\hline Iniciativa del medio & $11 \%$ & $20 \%$ & $27 \%$ \\
\hline Registro del medio & $26 \%$ & $22 \%$ & $26 \%$ \\
\hline Nota pagada & $3 \%$ & $3 \%$ & $1 \%$ \\
\hline Nota auto referencial & $4 \%$ & $0 \%$ & $1 \%$ \\
\hline
\end{tabular}

"Sinfuentismo" y "unifuentismo": sobre el uso de fuentes periodísticas

Si bien las noticias no llegan por sí solas a las redacciones, como se advertía en el apartado anterior, al periodista no le corresponde inventárselas. Es, esencialmente, la presencia de fuentes informativas lo que reviste de verdad el contenido de un noticiero.

Por ello, una gran alerta que se enciende, a la luz de la investigación del OM y el CM, es que de las 3857 notas analizadas de todos los telediarios, el 20\% de ellas (771 piezas informativas) no registran fuente alguna (ver gráfico 2); en otras palabras, es imposible para el televidente identificar quién dio la información. Como se puede observar (en la tabla 3), esta peligrosa práctica ( a la que se le llamado "sinfuentismo") es compartida casi en igualdad de condiciones en el plano nacional, regional y local.

\section{Gráfico 2. Uso de fuentes de información}

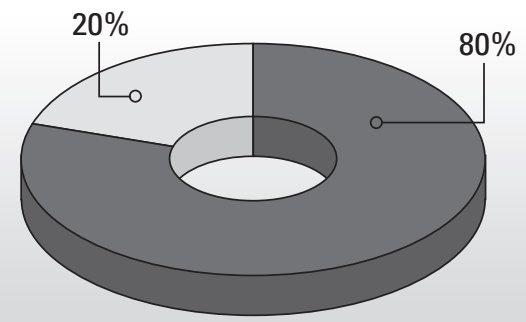

$\square$ Sin fuente $\quad \square$ Con fuente
Gráfico 3. Notas con un solo tipo de fuente

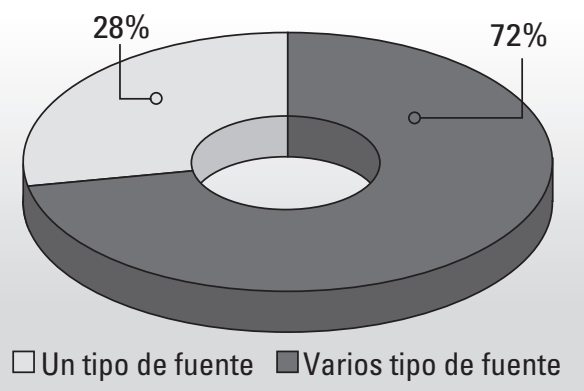

Tabla 3. Uso de fuentes de información

\begin{tabular}{|l|c|c|c|}
\hline \multicolumn{4}{|l|}{ Uso de fuentes de información } \\
\hline $\begin{array}{l}\text { Uso de } \\
\text { fuentes }\end{array}$ & $\begin{array}{c}\text { Noticieros } \\
\text { nacionales }\end{array}$ & $\begin{array}{c}\text { Noticieros } \\
\text { regionales }\end{array}$ & $\begin{array}{c}\text { Noticiero } \\
\text { local }\end{array}$ \\
\hline Sinfuentes & $28 \%$ & $8 \%$ & $3 \%$ \\
\hline Confuentes & $4 \%$ & $3 \%$ & $4 \%$ \\
\hline
\end{tabular}

Pero la nube negra que se cierne sobre la credibilidad de la información emitida por los diversos noticieros es todavía más alarmante: de las piezas que sí tienen fuentes (3.086), el 72\% evidencia la consulta de un solo tipo de ellas. Este fenómeno es mejor conocido como "unifuentismo" (ver gráfico 3).

Así, de las 6007 fuentes registradas en las 154 emisiones de noticieros estudiados, el $33 \%$ correspondían a fuentes testimoniales -entendidas como los testigos directos del hecho noticioso-; el 24\%, gubernamentales -poder ejecutivo y organismos adscritos-; el $16 \%$, fuentes oficiales privadas - persona o entidad del sector privado o la sociedad civil-y el 10\% eran fuentes oficiales públicas-rama legislativa y judicial-. Relegadas a una proporción ínfima, se encuentran las fuentes expertas - persona que opina en virtud de sus conocimientos- con un 5\% (ver gráfico 4). 


\section{Gráfico 4. Tipo de fuentes usadas para la} información

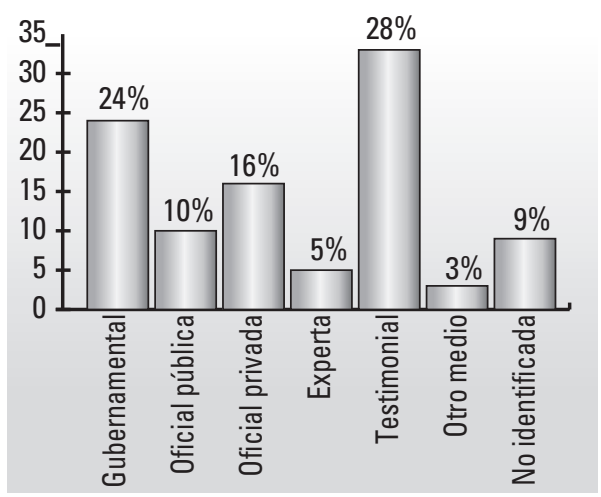

La jerarquización señalada en cuanto a tipo de fuentes, se mantiene tanto en los telediarios nacionales como regionales, no así en el caso de City Noticias o el informativo local (ver tabla 4). En éste, las fuentes testimoniales tienen un peso del $63 \%$, y ahí cabría sugerir la delicada posibilidad de que el periodista esté delegando su tarea en el ciudadano, bajo el fundamento de dar cabida al periodismo cívico, premisa errónea por completo.

Tabla 4. Tipo de fuentes usadas para la información

\begin{tabular}{|l|c|c|c|}
\hline \multicolumn{4}{|l|}{ Tipo de fuentes usadas para la información } \\
\hline Tipo & $\begin{array}{c}\text { Noticieros } \\
\text { nacionales }\end{array}$ & $\begin{array}{c}\text { Noticieros } \\
\text { regionales }\end{array}$ & $\begin{array}{c}\text { Noticiero } \\
\text { local }\end{array}$ \\
\hline Gubernamental & $25 \%$ & $27 \%$ & $12 \%$ \\
\hline Oficial pública & $14 \%$ & $7 \%$ & $7 \%$ \\
\hline Oficial privada & $17 \%$ & $19 \%$ & $6 \%$ \\
\hline Experta & $5 \%$ & $7 \%$ & $2 \%$ \\
\hline Testimonial & $24 \%$ & $30 \%$ & $63 \%$ \\
\hline Otro medio & $4 \%$ & $2 \%$ & $4 \%$ \\
\hline No identificada & $11 \%$ & $8 \%$ & $6 \%$ \\
\hline
\end{tabular}

Previo al cierre de este aparte debe destacarse otro dato: la presencia en un $9 \%$ de fuentes no identificadas, que aunque pareciera insignificante se traduce en 539 piezas informativas que no acreditaron con nombre propio el origen de su información, ocultaron la identidad del testigo ante la cámara o se refirieron a la fuente en términos generalistas como: "fuentes de alta credibilidad", "fuentes cercanas al gobierno" y "según algunos analistas", etc.

\section{Una sola postura, una sola "verdad": sobre el equilibrio} informativo

En el ejercicio periodístico no basta con tener muchas fuentes en una nota si sus posturas ideológicas son las mismas. En este caso, se genera un sesgo bien sea a favor o en contra del tema tratado. Se refuerza una idea que, no en pocas ocasiones, puede estar cargada de prejuicios sobre un suceso o sobre el protagonista de la información.

Al respecto, del total de los noticieros colombianos que fueron analizados, el 75\% de las notas tiene un solo punto de vista y un $11 \%$ más, muestra varios puntos de vista, pero con uno predominante, es decir, que se mantiene el sesgo. Tan solo en el 14\% de los casos existen varios puntos de vista que guardan equilibrio (ver gráfico 5).

\section{Gráfico 5. Equilibrio informativo}

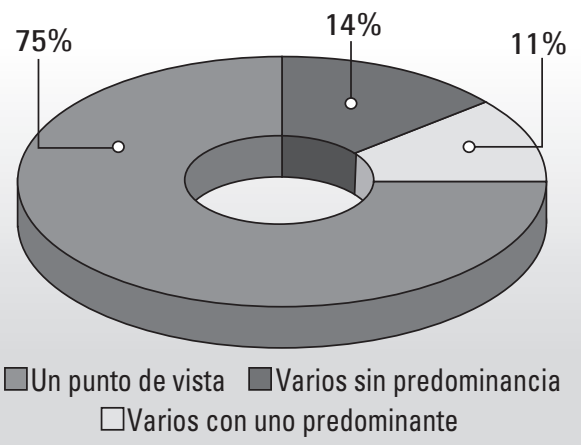

Desagregando los datos, salta a simple vista que la realidad referida es calcada por 
los medios nacionales que raramente utilizan (11\%) varios puntos de vista. Tal situación intenta ser rescatada por los noticieros regionales con 7 puntos porcentuales por encima (18\%), proporción que comparte el canal de Bogotá con el 17\%.

Al parecer no se comprende que "ante un hecho noticiable determinado, el (telediario) necesita disponer de varias fuentes contrastables para que su propia versión no sea una mera transcripción o reproducción de la que le ha proporcionado una sola fuente, ni refleje tan sólo la versión de una de las partes del conflicto" (Borrat, 1989).

En la tabla 5 se puede verificar la relación estricta entre el uso de fuentes y el grado de equilibrio informativo, ratificando que cualquier tipo de amparo consultado, en la mitad o en más de la mitad de los casos, hace parte de una pieza informativa que sólo ofrece un punto de vista o en su defecto que brinda varios puntos de vista pero con la predominancia de alguno de ellos.

Tabla 5. Equilibrio informativo

\begin{tabular}{|l|c|c|c|c|c|c|c|c|}
\hline \multirow{2}{*}{$\begin{array}{l}\text { Fuente de la } \\
\text { información }\end{array}$} & \multicolumn{4}{|c|}{$\begin{array}{c}\text { Un solo punto de } \\
\text { vista }\end{array}$} & \multicolumn{2}{|c|}{$\begin{array}{c}\text { Varios puntos con } \\
\text { uno predominante }\end{array}$} & $\begin{array}{c}\text { Varios puntos sin } \\
\text { uno predominante }\end{array}$ & \multicolumn{3}{|c|}{ Total } \\
\cline { 2 - 9 } & Frec. & $\%$ & Frec. & $\%$ & Frec. & $\%$ & Frec. & $\%$ \\
\hline Gubernamental & 697 & $62 \%$ & 209 & $18 \%$ & 225 & $20 \%$ & 1131 & $100 \%$ \\
\hline Oficial pública & 270 & $58 \%$ & 105 & $22 \%$ & 93 & $20 \%$ & 468 & $100 \%$ \\
\hline Oficial privada & 445 & $63 \%$ & 119 & $17 \%$ & 142 & $20 \%$ & 706 & $100 \%$ \\
\hline Experta & 173 & $71 \%$ & 19 & $8 \%$ & 53 & $22 \%$ & 245 & $100 \%$ \\
\hline Testimonial & 481 & $50 \%$ & 220 & $23 \%$ & 257 & $27 \%$ & 958 & $100 \%$ \\
\hline Otro medio & 146 & $84 \%$ & 11 & $6 \%$ & 17 & $10 \%$ & 174 & $100 \%$ \\
\hline No identificado & 203 & $54 \%$ & 81 & $21 \%$ & 93 & $25 \%$ & 377 & $100 \%$ \\
\hline
\end{tabular}

Fuente: Datos del estudio

Ahora, al hacerse el mismo ejercicio de revisar el número de puntos de vista en las piezas informativas de la franja prime time según su temática, se encontró un sesgo en las notas de entretenimiento, ya que ellas tienden a consultar solo una arista de la realidad (85\%). Otras noticias que ven comprometida su confiabilidad son las de índole internacional y deportivo que solo en el $9 \%$ y $3 \%$ de los casos, respectivamente, mencionan varios puntos de vista de manera equilibrada (ver tabla 6). 
Tabla 6

\begin{tabular}{|c|c|c|c|c|c|c|c|c|}
\hline \multirow{3}{*}{$\begin{array}{l}\text { Temática de la pieza } \\
\text { informativa }\end{array}$} & \multicolumn{4}{|c|}{ Equilibrio informativo } & & & & \\
\hline & \multicolumn{2}{|c|}{$\begin{array}{c}\text { Un solo punto de } \\
\text { vista }\end{array}$} & \multicolumn{2}{|c|}{$\begin{array}{l}\text { Varios puntos } \\
\text { de vista con uno } \\
\text { predominante }\end{array}$} & \multicolumn{2}{|c|}{$\begin{array}{c}\text { Varios puntos de vista } \\
\text { sin predominancia de } \\
\text { alguno }\end{array}$} & \multicolumn{2}{|c|}{ Total } \\
\hline & Frec. & $\%$ & Frec. & $\%$ & Frec. & $\%$ & Frec. & $\%$ \\
\hline Política & 344 & $69 \%$ & 76 & $15 \%$ & 79 & $16 \%$ & 499 & $100 \%$ \\
\hline Judicial & 447 & $76 \%$ & 74 & $13 \%$ & 65 & $11 \%$ & 586 & $100 \%$ \\
\hline Economía & 246 & $79 \%$ & 35 & $11 \%$ & 31 & $10 \%$ & 312 & $100 \%$ \\
\hline Deportes & 416 & $76 \%$ & 36 & $7 \%$ & 96 & $18 \%$ & 548 & $100 \%$ \\
\hline Entretenimiento & 429 & $85 \%$ & 17 & $3 \%$ & 58 & $12 \%$ & 504 & $100 \%$ \\
\hline Internacional & 213 & $76 \%$ & 25 & $9 \%$ & 42 & $15 \%$ & 280 & $100 \%$ \\
\hline Ciencia y tecnología & 33 & $77 \%$ & 6 & $14 \%$ & 4 & $9 \%$ & 43 & $100 \%$ \\
\hline Otros & 760 & $70 \%$ & 145 & $13 \%$ & 180 & $17 \%$ & 1085 & $100 \%$ \\
\hline
\end{tabular}

Fuente: Datos del estudio.

La tiranía de la superficialidad: sobre el uso de elementos de contexto

Para el periodismo de calidad, la información por sí sola no basta. Y ante esta premisa el OM y el CM hallaron que sólo alrededor de la mitad (53\%) de las notas periodísticas analizadas cuenta con información de contexto (ver gráfico 6).

\section{Gráfico 6. Presencia de elementos de contexto}

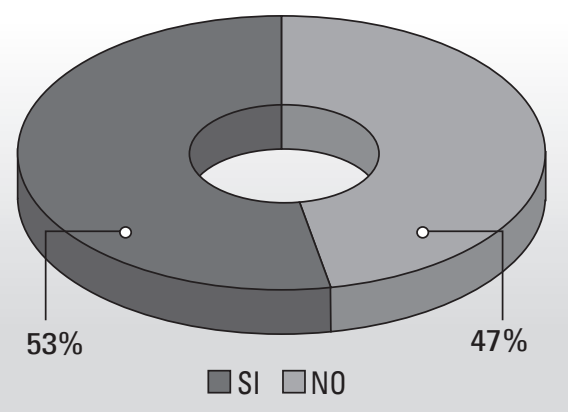

\section{Gráfico 7. Tipo de elementos de} contextualización

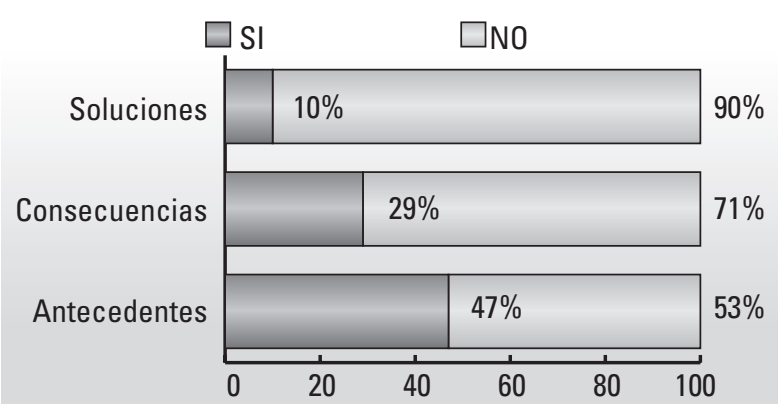

La noción de la complejidad natural de los acontecimientos cede terreno ante criterios como la sencillez y la rapidez de la comunicación televisada. Por esto cuando se contextualiza, no todos los elementos de referencia están presentes en los diferentes casos: un $47 \%$ incluye antecedentes; un $29 \%$ consecuencias y nada más que el $10 \%$ provee soluciones (ver gráfico 7). Los medios buscan así que la gente entienda la causa de los hechos, pero no están interesados en mostrar las repercusiones del evento y menos en aportar soluciones. 
Desde luego este análisis sería también simplista si no se advierte que los informativos son espacios para la proclamación de lo instantáneo, lo urgente y lo inmediato (Catela, 2005); cosa que el OM y el CM pudieron constatar:

Una pieza informativa, dentro de un noticiero colombiano, tiene una duración promedio de 76 segundos (1.2 minutos), según la investigación. Un tiempo adecuado, quizá, para las exigencias del lenguaje audiovisual, pero no apto para cumplir con la tarea de contextualización. Obviamente en tan corto lapso sólo hay oportunidad para dar respuesta al qué, quién, dónde y cuándo ( es decir, describir el "instante"), resultando imposible ofrecer el por qué y el para qué de los hechos.

Pero si se va más allá, el bajo nivel de contextualización en los noticieros colombianos podría tener otra explicación: el casi desaparecimiento, de los géneros mayores del periodismo como la crónica y el reportaje (ver gráfico 8), de los que se ha dicho que por su carácter interpretativo ofrecen mayores elementos referenciales.

Gráfico 8. Género de las piezas informativas

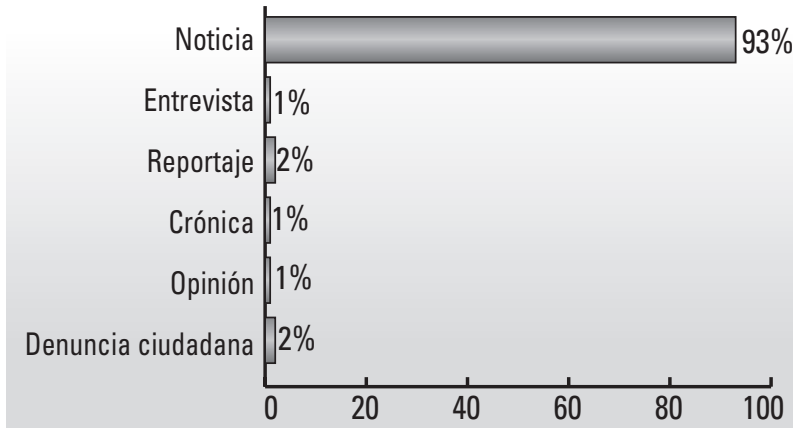

\section{Discusión y conclusiones}

Ignacio Ramonet (1999) atina al preguntarse: ¿qué aspectos dan valor a una infor- mación? A lo que responde: "es fácil comprobar que cuanto más cerca de la verdad está una información, más cara es, y cuanto más alejada está, más barata resulta”.

No obstante, luego de la revisión del OM y el CM al tratamiento dado por los telediarios colombianos a la información, en la franja prime time, queda la sensación de que las empresas mediáticas actuales no reconocen en la búsqueda de la verdad su mejor arma para competir en el mercado. De hacerlo, la investigación prosperaría como un compromiso inculcado al reportero en cada concejo de redacción; la consulta de fuentes diversas y el contraste entre las mismas sería una labor ineludible en cada jornada, tal como resultaría la entrega a la audiencia de mayores elementos de contexto que le permitan comprender más y mejor los hechos noticiosos.

Descalificada la verdad, la calidad de tipo moral a la que se hizo referencia en la introducción también queda vituperada. Y por lo mismo hacer de la calidad un diferencial de competitividad es una oportunidad desaprovechada por las empresas informativas colombianas. Así pues, las implicaciones de semejante descuido hacen que:

\section{Uno)}

El predominio de la iniciativa de la fuente a la hora del establecimiento de la agenda temática de un noticiero sea mayor, según lo evidenciado en la investigación, además puede leerse de esta manera: primero, para un medio es más rentable cubrir una información lista y gratuita que costear las necesidades de una unidad investigativa propia. Segundo, los periodistas han terminado por sucumbir ante uno de sus mayores verdugos, pero que contradictoriamente 
se enarbola como una de las aventajadas cualidades de los medios audiovisuales: la inmediatez. Ésta, definitivamente, exige tomar registro de lo primero que se tenga a mano (rueda de prensa, discurso parlamentario, etc.).

A ello hay que agregar que la televisión tiene lógicas propias de información. A veces el mayor y único criterio de noticiabilidad es la "imagen". Sólo lo que es digno de contarse en imágenes, es digno de ser noticia. Y en esto las fuentes interesadas sí que han avanzado a pasos agigantados, pues cuentan con sus propios gabinetes o equipos de prensa que se encargan de procesar la información y montar el "espectáculo" para que sea cubierto por los medios de comunicación.

El triunfo dela fuente como origen supremo de lainformación es, entonces, unindicador muy peligroso ya que desvirtúa el papel natural del periodista: elde mediador entrela realidad-ofrecida en estecaso por una persona o institución-y el público. Dicho rol es remplazado por la condición de simple vocero, y téngase en cuenta que "recopilary transcribir no es investigar... (y) pensar en el trabajo periodístico sin investigación equivaldríaaimaginar unabrújulasin cuadrantesalosqueapuntar”'(FOPEA, 2007).

En definitiva, "un medio de comunicación, cualquiera que sea su naturaleza, tendrá contenidos periodísticos de mayor calidad cuanto mayor sea la investigación cotidiana que aplica a la elaboración de sus contenidos" (FOPEA, 2007), puesto que no hay peor maldad contra el rigor periodístico, que la pereza del reportero de observar, objetar, deducir y dudar; pereza de buscar con creatividad y sugerir con originalidad.

\section{Dos)}

"La credibilidad de un medio está en relación directa con la cantidad, calidad y pluralismo de las informaciones y de las fuentes que cita", afirma Borrat, referido por López (1995:33). Un argumento que insinúa la necesidad no sólo de citar un testimonio sino de aludir a varios de ellos; se trata de una apuesta por la ponderación de las diferentes aristas de un suceso.

Empero, el ritmo desenfrenado de la producción de información en la época actual trae como consecuencia las perversas rutinas del sinfuentismo y el unifuentismo, según lo hallado en la investigación.

La real dimensión de lo que se ha decidido llamar "sinfuentismo", se advierte al atender lo dicho por Mar de Fontcuberta (1993): un medio sin fuentes es un medio muerto. Y a ello debe sumarse que "la imposibilidad de adjudicar la información a una persona o entidad concreta, deja abierta la duda de que ésta sea un simple rumor o un invento del periodista. La credibilidad queda seriamente comprometida en estas situaciones" (Gutiérrez-Coba, 2004: 9).

En tanto, el "unifuentismo" es darle cabida solamente a una voz o monopolizar el entendimiento de la realidad; es desconocer una premisa básica: ningún ser humano, por letrado que sea u ostente su condición como testigo directo, será capaz de dar razón del ciento por ciento de un hecho.

Claro, un balance justo obliga a indicar que en Colombia, al igual que en muchos países de la región y del mundo, las fuentes tienden a restringir el acceso a los periodistas sobre todo cuando sus preguntas le son incómodas a sus intereses.

Pero aquí de lo que se trata, en definitiva, es de señalar que, a veces, "los noticieros dedican más recursos a los ornamentos visuales que a cumplir el contrato mediático: pocas -o ninguna fuente-, insuficiente 
información y vaguedad en los datos" (Duplatt, s.f: 3).

Un estudio en perspectiva permite observar que, de acuerdo con lo analizado por el OM y el CM, la mitad de las fuentes (gubernamentales, públicas y privadas) están ligadas al poder político o económico del país. El problema del oficialismo ${ }^{2}$ en el periodismo no es algo nuevo; se han hecho advertencias desde diversas tribunas de la academia debido a la dañina percepción que de la realidad puede sembrarse en la audiencia.

"La excesiva dependencia de fuentes oficiales tiende a legitimar la estructura de poder en la sociedad y, en ocasiones, termina en la parcialización de la información y la desinformación" (Solosky, 1989: 69).

El uso de fuentes gubernamentales, obviamente, no puede satanizarse. Ellas, como cualquier otro tipo de testimonios, son válidas y necesarias; el problema radica en que se conviertan en el único recurso de validación informativa, además de la abismal diferencia que guardan frente a otras voces autorizadas como las fuentes expertas, cuya figuración debería ser mayor dada su capacidad para ofrecer al televidente análisis, explicación y elementos de contexto sobre cualquier hecho.

\section{Tres)}

Es obvio que cada una de las fuentes consultadas sobre un hecho (político, judicial, deportivo, etc.) tenga y quiera trasmitir su propia verdad. La tarea del reportero ante ello es asegurarle al ciudadano la entrega de todas y cada una de las "versiones" que puedan existir al alrededor del suceso, sin manipulación alguna, para que sea el espectador quien libremente sopese cada uno de los argumentos y se haga a un juicio propio.

Infortunadamente esta labor no ha sido asumida por los noticieros de la televisión abierta en Colombia. Se ha echado por tierra el requerimiento ético del equilibrio informativo, entendido como pluralidad de puntos de vista o variedad de ángulos desde los cuales se presenta la nota periodística.

Ya Dragnic (2007:37) advertía que "es difícil alcanzar un total Equilibrio Informativo. Pero sí es posible, a través de una política editorial independiente y una línea informativa precisa, alcanzar un nivel de equilibrio suficiente, para que los receptores puedan obtener informaciones no sesgadas ni manipuladas".

¿De qué manera el ciudadano-espectador tomará alguna decisión con respecto a la información difundida si la encuentra lejana a sus propias vivencias, dado que los hechos aparecen desconectados del ambiente que los rodea?

La única manera es que los noticieros superen la lógica macabra del discurso fragmentado, cosa que no han hecho en Colombia de acuerdo con la investigación. El reportero debería revisar y

2. "Entre todas las posibles fuentes de información, los periodistas tienden a favorecer las oficiales, debido a que están mejor equipadas para satisfacer las necesidades organizacionales de los reporteros. En efecto, las dependencias de los gobiernos federales, estatales o municipales cuentan con departamentos de comunicación social que facilitan la labor de los reporteros. Los atienden en los horarios adecuados a su jornada de trabajo, les proporcionan boletines, fotografías, entrevistas con los funcionarios. El hecho de que el funcionario ocupe un puesto político le da legitimidad para opinar, lo que evita que el reportero pierda tiempo en determinar si la fuente es creíble o no" (Lozano, 2007:29). 
buscar en la historia, hilar y conectar los diferentes sucesos de la coyuntura y hacer un seguimiento a sus relatos. Pero, sobre todo, el periodista tendría que apartarse de la premisa, como sugiere Ramonet (1999), de que para la audiencia el "ver" es suficiente para "comprender".

De lo que se trata es de contextuali$z a r^{3}$ la información, acto en el que para el escritor y periodista Ryszard Kapuscinski (2002) se puede diferenciar el buen del mal periodismo: en el buen periodismo, además de la descripción de un acontecimiento, se tiene también la explicación de por qué ha sucedido; en el mal periodismo, en cambio, se encuentra sólo la descripción, sin ninguna conexión o referencia al contexto histórico.

Muy bien le vendría a la televisión recordar que fuera de la noticia escueta, existen géneros "generosos", periodísticamente hablando, como la crónica y el reportaje que están para hacerle frente a la tiranía de la superficialidad y la prisa.

En conclusión, las empresas informativas colombianas están tirando por la borda la oportunidad de apropiarse de principios deontológicos para acreditarlos como valiosísimos activos intangibles. Por alguna razón desconocen la estrecha relación entre calidad y rentabilidad (Merrit, 1998; Meyer, 2004; Oyedeji y Duncan, 2006). Los directores y productores de los noticieros nacionales, regionales y local no aprecian que ofrecer noticias fieles a la verdad incide en el comportamiento del televidente, el cual "estará más dispuesto a consumir esas noticias, a pagar un mayor precio por ellas, y será un consumidor más leal (...) y en la medida que haya más demanda habrá más anunciantes, y por lo tanto, mayores ingresos" (Rastrollo, 2010:13).

Aunque parezca osado afirmarlo, el triunfo en el mundo de la oferta y la demanda está lejos del ofrecimiento nato de espectáculo y noticias light (fáciles y rápidas de consumir), y mucho más cerca del rigor en la selección de los temas, la elección de las fuentes y la sana interpretación de los hechos.

3. Galdón (1994: 161) asegura que "el dar la verdad necesaria contextualizada y explicada y con sentido significa que hay que situarla en un contexto espacio-temporal, apuntar su significación en el devenir histórico, suministrar una información centrada en el proceso y no en los acontecimientos aislados, prever mediante el conocimiento del pasado, el análisis del presente y la proyección en el futuro, tener en cuenta los procesos ideológicos relacionados con los acontecimientos; explicar el sentido que las cosas tienen por sí y por su repercusión en nosotros (...)". 


\section{Bibliografía}

1. Alessandri, F., Edwards, C., Pellegrini, S., Puente, S., Rozas, E., Saavedra, G. y Porta, W. (2001). VAP: un sistema métrico de la calidad periodística. Revista Cuadernos de la Información, 14. Santiago de Chile: Facultad de Comunicación de la Pontificia Universidad Católica de Chile (PUC). Disponible en: dialnet.unirioja.es/servlet/ fichero_articulo codigo $=2938296 \&$ orden $=0$. [Consultado el 5 de julio de 2010] .

2. Benito, A. (2001). Diccionario de Periodismo. Madrid: Acento.

3. Borrat, H. (1989). El periódico como actor político (propuesta para el análisis del periódico independiente de información general); tesis de Doctorado; Facultad de Ciencias de la Información, Universidad Autónoma de Barcelona.

4. Castells, M. (2000). La era de la información: economía, sociedad y cultura. Madrid: Alianza Editorial.

5. Catela, I. (2005). Ética de la Televisión. Consejos de sabios para la caja tonta. España: Desclée.

6. Dader, J. (2009). Periodismo en la hipermodernidad: consecuencias cívicas de una identidad débil (y algunas vías de reconstrucción). Publicado en Textual Visual Media, 2.

7. De Fontcuberta, M (1993). La noticia. Barcelona: Paidós.

8. Delbosco, P., Fabbro, G., Farré, M., Piscitelli, A. (2006). Calidad televisiva: tendencias y valores en la programación argentina. Buenos Aires: Universidad Austral, Facultad de Comunicación.

9. De la Torre, L., Téramo, M. (2007). Investigación sobre calidad periodística en La Argentina. En FOPEA (2007). Periodismo de calidad: debates y desafíos. Buenos Aires: La Crujía.

10. Duplatt, A. $(\mathrm{s}, \mathrm{f})$. Volver a las fuentes. Publicado en Actualidad Informativa. Universidad Nacional de la Patagonia. Disponible en: www.narrativas.com.ar/ Apuntes/Prensa\%20y\%20TV.doc. [Consultado el 14 de julio de de 2011]

11. Entrevista a Ignacio Ramonet. (1999). Ver no es comprender. Hecha por Txema García. Publicada en 7K, suplemento dominical de GARA. Disponible en: http:// www.baietz.org/zaharra/es/gogoeta/global/ramonet_ver_comprender.htm. [Consultado el 20 de junio de 2011].

12. Entrevista a Olga Dragnic. (2007). Hecha por Klibis Marín. Disponible en: www. revistalatinacs.org/_2008/alma02/95metodo_Klibis.pdf. [Consultada el $10 \mathrm{de}$ agosto de 2011]

13. FOPEA. (2007). Periodismo de calidad: debates y desafíos. Recopilado por Adriana Amado Suárez. Buenos Aires: La Crujía.

14. Galdón, G. (1994). Desinformación, métodos, aspectos y soluciones. Pamplona: Universidad de Navarra.

15. García, J. (1996). Periodismo de Calidad: estándares informativos en la CBS, NBC y $A B C$. Pamplona: Eunsa.

16. García, J. (2007). El infoentretenimiento en los informativos líderes de audiencia en la Unión Europea. Revista Análisi, 35. Universidad Autónoma de Barcelona.

17. Grijelmo, A. (2001). El estilo del periodista. Madrid: Santillana. 
18. Gouldner, A. (1976). The Dialectic of Ideology and Technology. New York: Oxford University Press.

19. Gutiérrez-Coba. L. (2004). "La justicia ignorada: tratamiento de la información judicial en la prensa”. Revista Palabra Clave, Número 10. Bogotá: Universidad de La Sabana.

20. Kapuscinski, R. (2002). Los cínicos no sirven para este oficio. Barcelona: Anagrama.

21. Lippmann, W. (1965). Public Opinion. New York: The Free Press.

22. López, M. (1995). Cómo se fabrican las noticias. Fuentes, selección y planificación. Barcelona: Paidós.

23. Lozano, J. (2007). Newsmaking y gatekeeping: cómo se producen las noticias. En Igartua, J. Medios de comunicación, inmigración y sociedad. Salamanca: Universidad de Salamanca Soria, C. (1989). La crisis de identidad del periodismo. Barcelona: Mitre.

24. Mattelart, A. (2002). Historia de la sociedad de la información. Barcelona: Ediciones Paidós Ibérica.

25. McCombs, M. y Evatt, D. (1995). Los temas y los aspectos: explorando una nueva dimensión de la Agenda-Setting. Comunicación y Sociedad, 8 (1). Disponible en: http://www.unav.es/fcom/comunicacionysociedad/en/articulo.php?art_id=169. [Consultado el 22 de julio de 2010].

26. McCombs, M. E. y Shaw, D. L. (1972). The agenda-setting function of mass media. Public Opinión Quarterly, 36.

27. Merritt, D. (1998). Public journalism and public life: why telling the news is not enough. 2nd ed. Mahwah, N.J.: Erlbaum.Mullainathan y Shleifer, 2003.

28. Meyer, P. (2004). The vanishing newspaper. Columbia, MO: University of Missouri Press.

29. Monedero, J. (2009). El gobierno de las palabras: política para tiempos de confusión. Madrid: Fondo de Cultura de España.

30. Oyedeji, T.; Duncan, F. (2006). The relationships among audience loyalty, perceived quality, and media credibility of cable news networks. Paper presented at the Annual convention of the Association for Education in Journalism and Mass Communication, San Francisco, CA.

31. Pérez, R. A. (2008). Estrategias de Comunicación. Barcelona: editorial Ariel.

32. M. A, Rastrollo. (2010). Credibilidad de la empresa informativa. Una perspectiva organizativa. Publicada en Revista Latina de Comunicación Social. Universidad de la Laguna. Disponible en: http://www.revistalatinacs.org/10SLCS/actas_2010/001Reig. pdf [consultado el 1 de septiembre de 2011].

33. Solosky, J. (1989). Sources and Channels of local News. En Journalism Quaterly, Vol. 66.

34. Tuggle, C. (2001). Selecting stories and starting to write. En Tuggle, Carr, Forrest y Huffman, S. Broadcast news handbook. New York: McGraw-Hill.

35. Yarce, J. (1992). Periodista ser o no ser. Chía: Universidad de La Sabana. 